American Journal of Pharmacology and Toxicology 2 (2): 26-29, 2007

ISSN 1557-4962

(C) 2007 Science Publications

\title{
Evaluation of Local Injection of $0.5 \%$ Bupivacaine + 1/200000 Epinephrine for Post Tonsillectomy Pain Reduction
}

\author{
Keramat Mozafarinia and Maryam Amizadeh \\ Department of Ear, Nose, Throat, Kerman Medical Sciences University, Kerman, Iran
}

\begin{abstract}
To determine the effectiveness of postoperative local infiltration of Bupivacaine in decreasing post tonsillectomy pain. A double blind randomized clinical trail was conducted. Treatment group was

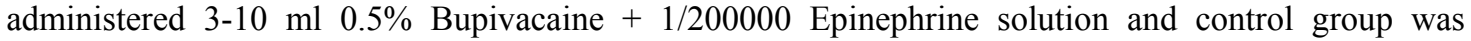
received 3-10 ml normal saline, locally. Outcome was post surgical pain, which measured by visual analogue scale (VAS), after $6,12,18$ hours post surgical. The mean scores of pain after surgery were lower grade in treatment group than placebo group. Post tonsillectomy pain suffered patients and required to reduce; hence pharmacotherapy seems necessary for it. Current finding was shown local $0.5 \%$ Bupivacaine $+1 / 200000$ Epinephrine injection is good for pain relief.
\end{abstract}

Key words: Tonsillectomy pain, infiltration of bupivacaine, effectiveness

\section{INTRODUCTION}

Tonsillectomy is one of the most commonly performed surgical procedures and is often associated with significant postoperative pain ${ }^{[1]}$.

Multiple techniques have been tried to reduce associated pain tonsillectomy. Some studies have shown pain severity is independent of surgical technique. In adult's postoperative pain, seems to be more than children ${ }^{[2,3]}$.

Children often become dehydrated, sometimes requiring readmission for intravenous hydration therapy and adults tend to remain home from work for a considerable length of time because of post tonsillectomy pain ${ }^{[4,5]}$.

Surgeons have also tried to seal the surgical wound and to cover exposed sensory nerves to reduce pain. Freeman et $a l .{ }^{[6]}$ obtained encouraging results using sucraifate in adult patienns. More recently, Stockli et $a l^{[7]}$ tried to cover the surgical wound with fibrin to reduce pain, but reported no benefit at all using this technique.

As far as local anesthetics are concerned, a few studies have been undertaken, with mixed results, in one of the earlier studies Violaris and Tuffin applied topical bupivacaine to the surgical wounds of adult patients and compared it with application of normal saline. They reported that the side treated with normal saline fared better, with less pain, than the side coated with $0.5 \%$ bupivacaine ${ }^{[8]}$. Jebeles et al. ${ }^{[4]}$ infiltrated fosse of children with pupivacaine and reported a marked reduction in pain after tonsillectomy even beyond the first week after surgery. However, show on advantage of bupivacaine injection over saline beyond the immediate post operative period ${ }^{[9,10]}$.

The purpose of this investigation was to determine the effectiveness of post operative local $0.5 \%$ bupivacaine $+1 / 200000$ epinephrine infiltration in post - tonsillectomy pain reduction.

\section{MATERIALS AND METHODS}

This double blined, randomized clinical trial was conducted at the Kerman University of medical sciences and health services, Shafa hospital Kerman, Iran from 2003 to 2004. One-handred twenty patients, 6-40 years under going tonsillectomy were enrolled in the study. They were devided to two equal groups, randomly.

Patients in the study group were injected with 3-10 $\mathrm{ml}$ of $0.5 \%$ bupivacaine $+1 / 200000$ epinephrine at the end to tonsillectomy and the patients in the control group were injected of 3-10 $\mathrm{ml}$ of normal saline. The site of injection was lateral base of uvula, postrosuperior and inferior of tonsilar fossa, middle third of pillars bilatarsbilars bilaterally (avoid to lateral pharyngeal wall injection, because of the danger of RIN paralysis and air way. Obstruction).

Corresponding Author: $\quad$ Keramat Mozafarinia, Department of Ear, Nose, Throat, Kerman Medical Sciences University, Kerman, Iran, Tel: 00989133439261 
Table 1: Characteristics of patients

\begin{tabular}{llllll}
\hline & & Control Group & Case Group & $\chi^{2}$ & P \\
\hline Age (year) & $<12$ years & $25(50 \%)$ & $25(50 \%)$ & 1 & 1.000 \\
Sex & $>12$ years & $35(50 \%)$ & $35(50 \%)$ & 3.943 & 0.054 \\
& Male & $34(\% 59.65)$ & $23(42.8 \%)$ & & 0.039 \\
Tonsilar & Female & $26(\% 41.27)$ & $37(58.73 \%)$ & & 0.981 \\
Grade & I & $7(41.2 \%)$ & $10(58.8 \%)$ & \\
Hypertrophy & II & $24(53.3 \%)$ & $21(46.7 \%)$ & \\
& III & $24(49 \%)$ & $25(51.1 \%)$ & \\
\hline
\end{tabular}

Results were randomly assigned to either the study group or control group and the surgeon were not aware of the identity of injected. The patients were given a form to complete after $6,12,18$ hours from the end of operation.

They were asked to mark the severity of their pain from $0-10$ ( 0 presenting no pain at a' 11 and 10 the worst pain) and keep records before and after their oral feeding. Subjective was assessed by visual analogue scale (VAS) and objective by dilution.

The patients did not know he identity of the injected solution they received. All patients (120) in this study underwent tonsillectomy under standardized general anesthesia in same technique. All patients received acetaminophen $15-30 \mathrm{mI} / \mathrm{kg}$. The data were analyzed for 2 samples with equal variances to calculate $P$ values.

\section{RESULTS}

Sixty-three females and 57 males for a total of 120 patients (age 6-40 years) entered the study. There were no statistically significant differences between the groups for sex, age, indication for surgery and tonsil grade for hypertrophy (Table 1).

Two types of pain (constant incisional pain and pain caused by drinking $50-100 \mathrm{ml}$ of water) were assessed with a visual analogue scale (VAS), 6, 12, 18 hours after tonsillectomy.

According to (VAS) results, in study group, the severity of pain was less than control group and also the group of patients (study group) infected with $0.5 \%$ bupivacaine $+1 / 200000$ epinephrine fared better and had less pain than saline group after oral liquid intake (Fig. 1 and 2). It was important that patients older than 12 years old had less pain than under 12 years individuals.

\section{DISCUSSION}

Tonsillectomy is a commonly performed procedure in children. Postoperative morbidity, including pain,

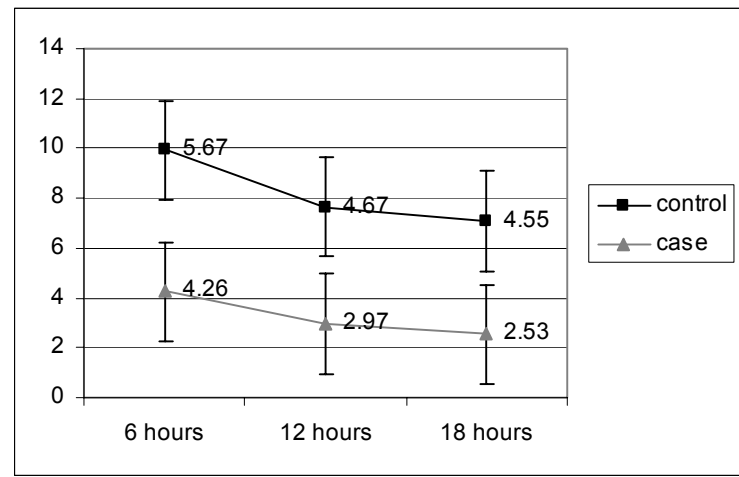

Fig. 1: Comparison of pain score after surgery

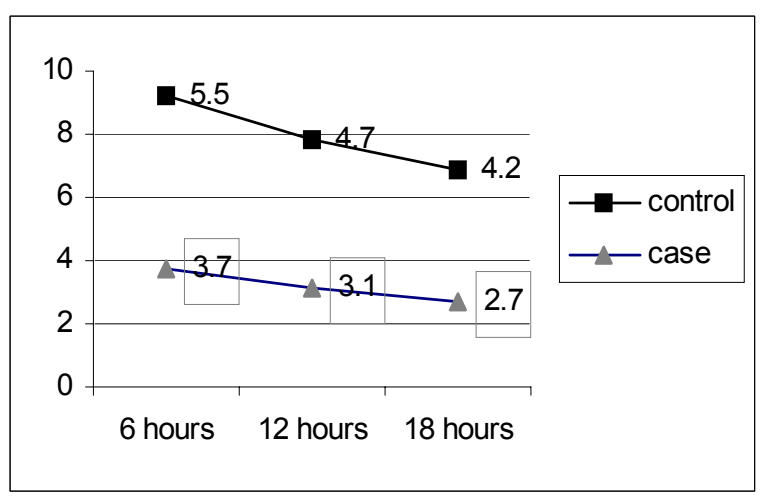

Fig. 2: Comparison of pain score after oral intake liquid

inadequate oral intake, dehydration and bleeding can create problems in the pediatric patient. Since postoperative pain often results in poor oral intake, many attempts have attempts have been to decrease the pain associated with tonsillectomy ${ }^{[11]}$.

Pain is a subjective and complex expression ${ }^{[12]}$. Its terms of measuement and standardization depend on personal experience, social and ethnic factors, perceptual abilites, anxiety level and the ability to describe the type and degree of pain based on some frame of reference ${ }^{[10]}$. It has been suggested that the pain after tonsillectomy is caused by inflammation, 
nerve irritation and spasm of exposed pharyngeal muscles. The pain does not completely subside until the muscle becomes covered with mucosa 14- 20 days after surgery ${ }^{[6]}$. Mary strategies have been proposed to eliminate or minimize the factors that result in postoperative pain. The surgical technique can be altered. Leach et $a l^{[13]}$ have compared the sharp dissection- snare technique with electrocautery. The electrocautery may cause more pain than sharp dissection alone. The dissection and snare technique was used exclusively in this study. Studies have shown than anti-inflammatory analgesics such as aspirin and indomethacin can decrease postoperative pain in patients undergoing tonsillectomy. The dissection effect seems to be related to the anti-inflammatory effect ${ }^{[1]}$. However, these agents are also strong inhibitors of prostaglandin's and platelets and can increase the incidence of postoperative bleeding ${ }^{[14]}$.

The concept that local infiltration of the operative area with a local anesthetic agents coats could alleviate postoperative pain has been known since the beginning of the century ${ }^{[15,16]}$. Local anesthetics have been injected into the tonsillar fossa to provide long- acting analgesia; Jebeles et $a l .{ }^{[4]}$ found that bupivacaine hydrochloride infiltration decreased post-tonsillectomy pain in a small group of patients. Johansen et al. ${ }^{[17]}$, found a significant effect in the VAS score for the period as whole after preincisional with bupivacaine: Broad man et al. ${ }^{[2]}$ and Schoem et al. ${ }^{[10]}$ found no important difference in postoperative pain with the use of peritonsillar infiltration of bupivacaine. In our study, after bupivacaine $+1 / 200.000$ epinephrine had been infiltrated in the first post-operative $6,12,18$ hours the pain decreased significantly when it was compared with the normal saline group $(\mathrm{p}<0.05)$ (Fig. 1 and 2). And also, in spite of other studies we found that patients older than 12 years old had less pain than 6-12 years group.

\section{CONCLUSION}

Postoperative pain is significant problem in patties who undergo tonsillectomy. Pain may lead to decreased oral intake and dehydration, lengthening hospital stay, increasing patient and parent anxiety and missing days of work in adults. According to results found in our study, post-tonsillectomy infiltration with $0.5 \%$ bupivacaine $+1 / 200,000$ epinephrine:

* Is a safe medication

* Reduces immediate and the first 18 hours postoperative poin in both children and adult population.
* Offers more advantage in control of posttonsillectomy pain in adults than children.

* Decreases the post-tonsillectomy oral feeding time.

* Post- tonsillectomy pain is not related to the reason of tonsillectomy, sex, age and tonsilar grade hypertrophy.

\section{REFERENCES}

1. Broadman, L.M., R.I. Patel and B.A. Feldman et al., 1989. The effects of peritonsillar infiltration on the reduction of intraoperative blood loss and post tonsillectomy pain in children, Laryngoscope, 99: 578-581.

2. Akkielan, A., A. Kalan and G.S. Kenyon, 1997. Diathermy tonsillectomy: Comparison of morbidity following bipolar and monople microdissction needle excision. J. Laryngol. Otol., 111: 735-738.

3. Kujawski, O., P. Dulguerov and C. Gysin et al., 1997. Microscopic tonsillectomy: A double-blind randomized trial. Otolarygol. Head Neck Surg., 117: 641-647.

4. Jebeles, J.A., J.A. Reilly and J.F. Gutierrez et al., 1991. The effect of pre-incisional infiltration of tonsils with bupivacine on the pain following tonsillectomy under general anesthesia. Pain, 47: 305-308.

5. Johes, J., S.D. Handler and M. Gurttnpian et al., 1990. The efficacy in children. Arch. Otolaryngol. Head Neck Surg., 16: 590-593.

6. Freeman, S.B. and J.K. Markwell, 1992. Sucralfate in alleviating post-tonsillectomy pain. Laryngoscope, 102: 1242-1246.

7. Stoeckli, S.J., K.S. Mor and A. Huber et al., 1999. A prospective randomized double- blind trial of fibrin glue for pain and bleeding after tonsillectomy. Laryngoscope, 109: 652-655.

8. Violaris, N.S. and J.R. Tuffin, 1989. Can posttonsillectomy pain be reduced by topical bupivacaine: Doudle blind controlled trial. J. Laryngol. Otol., 103: 592-593.

9. Telian, S.A., S.D. Handler and G.R. Fleisher et al., 1991. The effect of antibiotic therapy on recovery after tonsillectomy in children: A controlled study. Arch. Otolaryngol. Head Neck Surg., 17: 946-952.

10. Schoem, S.R., G.L. Eatkins and J.J. Kuhn et al., 1993. Control of early postoperative pain with bypivacaine in adult local tonsillectomy. Arch. Otolarygol. Head Neck Surg., 119: 292-293.

11. Ohlms, L.A., R.T. Wilder and B. Weston, 1995. Use of intraopetative corticosteroids in pediatric pediatric tonsillectomy. Arch., Otolaryngol. Head Neck Surg., 121: 737-742. 
12. Garron, D.C. and F. Leavitt, 1979. Demographic and affective covariates of pain. Psychosom. Med., 41: 525-534.

13. Leach, J., S. Manning and S. Schaefer, 1993. Comparison of two methods of tonsillectomy. Laryngoscope, 103: 619-622.

14. Carrick, D.G., 1984. Salicylates and posttosillectomy heamor rhage. J. Laryngol, Otol., 98: 803-805.

15. Goldsher, M., L. Podochin and M. Fradis et al., 1996. Effect of peritonsillar infilteration on posttonsillectomy pain. Ann. Otol. Rhinol. Layrngol., 105: 868-870.
16. Jebeles, J.A., J.A. Reilly and J.F. Gutierres et al., 1993. Tonsillectomy and adenoidectomy pain reduction by local bupivacaine infiltration in children. Int. J. Pediatr. Otorhinolaryngol., 25: 149154.

17. Johansen, M., G. Harbo and P. Illum, 1996. Preincisional infiltration with bupivacaine in tonsillectomy. Arch. Otolaryngol. Head Neck Surg., 122: 261-263. 